\title{
Multi-Criteria Optimization of Access Selection to Improve the Quality of Experience in Heterogeneous Wireless Access Networks
}

\author{
Quoc-Thinh Nguyen-Vuong, Member, IEEE, Nazim Agoulmine, Senior Member, IEEE, El Hadi Cherkaoui, \\ Member, IEEE and Laura Toni, Member, IEEE
}

\begin{abstract}
Wireless technology is one of the revolutionary advancements providing users with ubiquitous data and telephony access anywhere and anytime without any physical connection. The nowadays deployed wireless networks named WiFi, Worldwide Interoperability for Microwave Access (WiMAX), Universal Mobile Telecommunications Systems (UMTS) and Long Term Evolution systems (LTE) have different characteristics that make them complementary in term of performance, coverage and cost. This network variety presents an opportunity to provide better services to the end-users given the advances in mobile terminals. To reach this goal, an appropriate automatic network selection (ANS) mechanism, able to always select the best access network, is needed. This consists on constantly monitoring any type of available access networks, automatically selecting and switching to the best one, as the network that maximizes the users quality of experience taking into account their preferences as well as the terminal and network conditions. ANS is a multi dimension decision-making problem which can be solved by finding an appropriate complex trade-off between possibly conflicting criteria. In this paper, we propose an analytical model to capture the preferences of end-users. Based on this model, we design an ANS mechanism that takes into account all aspects of the trade-off between the quality of the connections, the preferences of the end users and the cost. To highlight the benefits of our approach from the perspectives of both end-users and network operator, we have implemented and tested the solution in a multi technologies simulator. Results show that the proposed solution outperforms the main stream approaches.
\end{abstract}

\section{INTRODUCTION}

With the advances in multiple access networks and multi mode terminals, end users are able to connect to any available wireless network such as Global System for Mobile Communications (GSM), General Packet Radio Service (GPRS), Universal Mobile Telecommunications Systems (UMTS), Wireless Local Area Network (WLAN), and Long Term Evolution (LTE) systems and Worldwide Interoperability for Microwave Access (WiMAX). These multi-mode terminals can be either equipped with multiple radio interfaces or with one single

Part of this work has been presented at the IEEE/IFIP Network Operations and Management Symposium Workshops (NOMS 2008 Conference) and at the IFIP/IEEE International Symposium on Integrated Network Management (IM 2011). T. Nguyen is with France Telecom- Orange, FRANCE. (email: thinh.nguyen@polytechnique.org). N. Agoulmine is with LRSM IBISC Lab. University of Evry Val d'Essonne, Evry, FRANCE. (email: nazim.agoulminedieee.org). E.H. Cherkaoui is with the Italian Institute of Technology (IIT), Via Morego 30, 16163 Genova, ITALY (email: elhadi.cherkaoui@iit.it). L. Toni is with École Polytechnique Fédérale de Lausanne (EPFL), Signal Processing Laboratory - LTS4, CH-1015 Lausanne, Switzerland (e-mail: laura.toni depfl.ch). reconfigurable interface [1] capable to communicate using any existing wireless access network protocol.

This evolution towards the next generation of wireless networks has led to an interesting paradigm shift where the user is no more passive but could influence the selection of the wireless access network. More precisely, Mobile Terminals (MTs) such as smartphones will not be fully controlled by the operators but will be able to select by themselves the best wireless access network that better satisfies end-user's preferences. By smartly exploiting this network diversity and the interworking between wireless access network technologies, the end-users Quality of Experience (QoE) can be significantly improved.

In this context, the automatic network selection (ANS) mechanism, a key mechanism that needs to be implemented in multi-mode terminals, is primordial. In traditional homogeneous networks, network selection is only based on signal quality from serving and neighboring access nodes, like Received Signal Strength (RSS) or Signal-to-Interference-plusNoise Ratio (SINR), and it is fully controlled by the network in the case of cellular systems. In heterogeneous networks and universal access facilities, ANS is a multi dimensions decision-making problem that involves a set of network and terminal parameters and complex trade-off between possibly heterogeneous criteria (e.g performance or cost) that could be defined by the end-user. Satisfying all criteria at the same time is proved to be difficult as some criteria may be in conflict. For example, some users may prefer the cheapest access network (e.g., teenagers) while others may prefer the highest data rate (e.g. professionals).

Lately, a variety of access network characteristics have been identified as potential network selection criteria [2][8]. However, to the best of our knowledge, a holistic study on how these criteria are used to take the right decision is still missing. With this work we aim at covering this step, focusing on the "pay-off" model of access network selection (i.e., decision metric and strategies of end-users and network operators), which is recognized to be a complex problem [9].

Among the different approaches addressing the multicriteria decision problem provided in the literature, in this work we consider the multi-criteria utility theory approach introduced by Keeney and Raiffa in [10]. Even though the utility function has been successfully used in the area of networking to capture the end-users criterion in decision making (e.g., power control in wireless cellular systems [11], 
radio resources management in wired and wireless networks [12], [13], as well as network selection [5]), calculating singlecriterion utility as well as aggregate utility remains relatively complex. Our goal is to propose an appropriate utility model with respect to the specificities of access network selection problem. Within this framework, user's requirements can be translated into relative weights that a user assigns to each criterion (e.g., price, quality, mobility, etc). In this way, the end-user is introduced in the control loop of the system, while the concept of fairness is subjective to each user. Some of them might prefer reducing the cost while others might be more interested in achieving the best quality. By introducing weights that are related to the preferences of the end-users, the equilibrium point is the one that better satisfies each subscriber from his own perspective and not from an arbitrary/common point of view.

Once the criteria are identified and the preferences are fixed, it is necessary to define a mechanism which allows the terminal to evaluate candidate access networks in order to identify the most suitable one given the user preferences. Therefore, the research questions we aim at responding are:

1) Is it possible to design a utility function able to capture the user preferences and sensitivity to a set of identified criteria?

2) From this model, is it possible to properly design an aggregate function that allows to build an efficient decision-making mechanism for ANS?

3) In a representative scenario with heterogeneous wireless networks and numerous mobile subscribers with different preferences, does the proposed solution performs as expected, i.e., subscribers do have a better experience with the proposed ANS compare to existing approaches?

4) Finally, how does the implementation of this solution in future terminals impact the business of wireless network operators?

To answer these questions, first we provide a through study of the required conditions on the utility functions. We show in particular why conventional additive criteria cannot guarantee the imposed conditions for network selection and we therefore propose novel single-criterion and multiple-criteria utility functions. Then, in order to evaluate the network performance from a theoretical point of view and later from a more realistic point of view, we provide both numerical and network simulations. The latter have been achieved by setting up a complete a simulation platform able to simulate the coexistence of all the technologies considered in our work. Results show that our proposed solution outperforms existing ones from different perspectives.

The remainder of the paper is organized as follows. In Section II, a summary of existing studies on access network selection techniques is presented. In Section III, we present the various existing wireless technologies and mobility issues and we present the overall system architecture environment. In Section IV, we describe the existing single-criterion utility functions, highlighting the main limitations. Based on these considerations, we propose a novel and more appropriate utility function. Analogously, in Section V, from the study of the existing multi-criteria functions, we highlight the fundamental properties that a suitable utility function should exhibit. Then, we propose our multi-criteria utility function for ANS. In Section VI, we explain how the proposed utility functions are integrated in the ANS mechanism. Further, we describe how the ANS is integrated in the MT and how it interacts with other components of the terminal. Section VII presents the components of the built simulator and the considered parameters and metrics to evaluate the proposed solution. Section VIII and Section IX present the numerical results as well as the simulation results of the proposed ANS. Finally, conclusions are provided in Section X.

\section{RELATED WORKS}

The problem of wireless access network selection and the Vertical HandOver (VHO) have been previously addressed in several contributions. The common goal of all approaches was to maintain the MT connection during the mobility of the user across heterogeneous and/or multi-operators access networks (e.g., walking, using a car, traveling in a train). To ensure a seamless handover, several mechanisms have been proposed, and can be classified in three different categories depending on the entity that controls the handover namely: Terminal Controlled Handover (TCH), Network Controlled Handover $(\mathrm{NCH})$ and hybrid Controlled Handover $(\mathrm{HCH})$.

For a comprehensive survey of these VHO algorithms, we refer the reader to [14] and [15]. In the following, we review the most relevant works from the literature, highlighting the key differences with our work. We first provide an overview on the access network selection in VHO algorithms, then we mention the works focused on general system architecture.

In [2], the authors proposed a TCH policy-based associated to a logarithmic cost function to identify the optimal time to initiate the VHO and to select the most appropriate interface. Unfortunately, the used cost function can not properly handle complex situations such as free access wireless networks (e.g. free $\mathrm{WiFi}$ hotspot). In the presence of an open wireless access network, the proposed ANS function will always select it even if its quality is very poor. In [3], a so called "Smart Decision Model" has been proposed to perform VHO among available network interfaces, by integrating user preferences and network related information. The proposed mechanism evaluates for each interface an additive multi-criteria cost function and the one with the highest score is selected. The idea behind this proposal is close to our solution, however the used additive multi-criteria cost function in [3] has critical limitations that are highlighted in Sec. V-A.

In [16], the authors proposed a VHO decision algorithm that takes into account not only network parameters but also the energy consumption to maximize the collective battery lifetime of MTs. The solution consists on resolving a multi-criteria optimization problem where the cost function encompasses the collective battery lifetime of MTs and the load balancing over access points (APs)/base stations (BSs). This decision is taken by the Vertical Handoff Decision Controller (VHDC) for all multiple overlapping networks covered by APs and/or BSs. The VHDC selects the best network among multiple 
overlapping networks (covered by APs or BSs), based on a set of optimization criterion and the cost function. However, this solution can be classified as a NCH since it necessitates some modifications in the core network to deploy the VHDC. Moreover, user criteria such as monetary cost, mobility model, and the weighted preferences of each criterion are not considered in the VHDC cost function.

Our work is orthogonal to this approach, proposing a mechanism which is implemented only in the terminal (TCH) and extending significantly the decision mechanism.

In parallel to these contributions addressing handover (HO) mechanisms, other works have focused on the architectures to support these mechanisms. For example, in the work presented in [17]-[20], a Media Independent Handover (MIH) mechanism proposed by the National Institute of Science and Technology (NIST) is presented as part of a joint work between IEEE 802.21 and Internet Engineering Task Force (IETF). The corresponding implemented software package has been used by several research groups including ourselves. MIH mechanism is a very important contribution since it isolats ANS mechanisms from mechanisms to gather information from the networks. In [21], [22] the authors evaluated the performance of a VHO scheme between 802.11 and 802.16 in terms of signaling cost, handover delay, packet loss and QoS. The authors considered an interworking architecture of wireless mesh backbone and proposed a vertical handoff scheme between 802.11 and 802.16 wireless access networks. Unfortunately, cellular network technologies were not considered. Finally, in [23], authors provided a comprehensive review of the literature on mobility management architectures for seamless handover of mobile users and provide directions for further works in this field highlighting the mandatory requirements for future architectures. Authors also proposed a new cross-layer architecture called Context-Aware Mobility Management System (CAMMS) which is an interactive approach to seamless handover of users and services but it is only a framework without detailed mechanisms.

In general, all these related works have been a source of inspiration for our contribution and more particularly the contributions from NIST [16]. The varieties of proposed VHO mechanisms are multiple and introduce several single-criterion and multiple criteria decision-making functions. However, while dealing with multi-criteria optimization in the context of wireless access networks, it is not only important to take into account the network properties in the decision-making but also their impact on the end user satisfaction. From this perspective, our contribution is innovative w.r.t. TCH. Regarding $\mathrm{NCH}$ and $\mathrm{HCH}$, our solution is actually orthogonal to them since it does not require any change in the core network.

\section{Overall ARCHITECTURE}

We now describe the general framework considered in this work and we highlight the importance of the ANS mechanism in dynamic networks. First, the investigated scenario is detailed, showing the coexistence of heterogeneous wireless network technologies. Then, we show why an opportunistic ANS mechanism is a crucial element in this dynamic environment. Finally, we describe the main metrics that have been

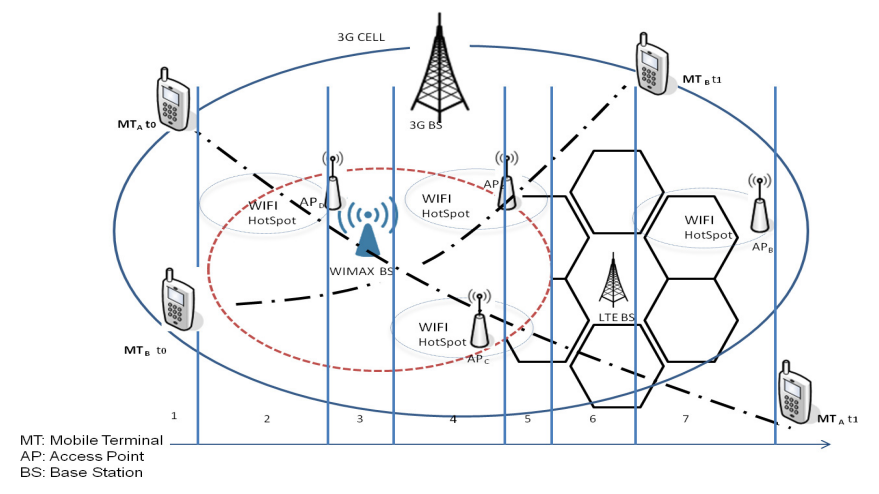

Figure 1. Heterogeneous wireless network diversity.

taken into consideration in the selection of the best access network.

\section{A. Considered Scenario}

We consider a scenario in which several wireless network technologies are deployed: IEEE 802.11 WLAN, IEEE 802.16 WiMAX, 3G and LTE. We consider an area that is fully covered by the $3 \mathrm{G}$ signal and partially by other technologies. Table I highlights the the characteristics of each technology. WiFi APs cover only limited zones while WiMAX, UMTS and LTE BS cover much larger zones. As shown in Fig. 1, the zones covered by heterogenous technologies are overlapped.

Within this area, we initially assume $N$ active users randomly distributed within the $3 \mathrm{G}$ cell. Each user, equipped with MTs, is able to move at random velocities following random paths, as described in Sec. VII-E. This means that users can be almost static (pedestrians moving at low velocity) or dynamic (users moving at high speed with any possible transportation). We also assume that users can start or end a connection at anytime and therefore the number of active users can randomly change during time. Thus, users might move throughout areas covered by heterogeneous wireless access networks, and it is of the MT benefit to find the best access network anytime and anywhere.

\section{B. Managing Mobility}

Fig. 1 depicts an example of the considered environment where a user A equipped with $M T_{A}$ is moving along a path where several access networks are deployed. The arrow at the bottom of the figure shows the different covered zones crossed by the MT. The terminal may select the best access network based on the user's preferences, the considered application, and the network status. In zone 1, the MT has a default selection, being the $3 \mathrm{G}$ cell the only available network. However, as the user moves along the path, the terminal crosses areas served by several networks.

During this movement, the MTs receive IEEE 802.21 control messages (that will be introduced later), including information about available access networks, link layer conditions as well as other useful information. For instance, in the area 2, the MT senses both $3 \mathrm{G}$ and LTE, while in the zone 3 it senses also the WiMAX network. The ANS mechanism implemented 
Table I

ACCESS NETWORKS CHARACTERISTICS

\begin{tabular}{|l||l|l|l|l|}
\hline Characteristic & WiFi & WiMAX & LTE \\
\hline \hline $\begin{array}{l}\text { Coverage area/Cellule } \\
\text { Size }\end{array}$ & $50 \mathrm{~m}-100 \mathrm{~m}$ & $500 \mathrm{~m}-1000 \mathrm{~m}$ & $500 \mathrm{~m}-1000 \mathrm{~m}$ \\
\hline $\begin{array}{l}\text { Trigger Link Going Down } \\
\text { factor }\end{array}$ & 1.2 & 1.1 & $\begin{array}{l}\text { IEEE802.21 is not imple- } \\
\text { mented }\end{array}$ & $\begin{array}{l}\text { Trigger Link Going Down } \\
\text { is not used }\end{array}$ \\
\hline $\begin{array}{l}\text { PHY Spec. Propagation } \\
\text { model }\end{array}$ & Two-Ray Ground Model & $\begin{array}{l}\text { OFDM and Two-Ray } \\
\text { Ground Model }\end{array}$ & $\begin{array}{l}\text { OFDMA/MIMO/SC- } \\
\text { FDMA }\end{array}$ \\
\hline Antenna Type & OmniAntenna & OmniAntenna & OmniAntenna \\
\hline Frequency Range & $2.4 \mathrm{Ghz}$ & $3.5 \mathrm{Ghz}$ & $2 \mathrm{Ghz}$ \\
\hline RX Threshold & $5.2 \times 10^{-10} \mathrm{~W}$ & $1.2 \times 10^{-9} \mathrm{~W}$ & $\begin{array}{l}\text { HHO selection based on } \\
\text { position }\end{array}$ & $1.0 \times 10^{-16} \mathrm{~W}$ \\
\hline Peak Data Rate (DL) & $11 \mathrm{Mbps}$ & $54 \mathrm{Mbps}$ & $100 \mathrm{Mbps}$ \\
\hline
\end{tabular}

in the MT periodically evaluates the value of each access network (this concept will be introduced latter in the paper) and identifies the best one. Therefore, the MT initiates the handoff to this network. If the handover fails, the second best available network is selected.

The above example highlights the importance of ANS mechanism to ensure the end users the always best connected service in heterogeneous networks. In this work, the ANS mechanism we aim to design is a TCH ANS mechanism as part of the MT operating system. To facilitate the introduction of such mechanism, we suppose that some high level media independent handover management functions such as the $\mathrm{MIH}$ framework (defined by the IEEE 802.21 standardization group) [24] are deployed in all wireless access networks and provide the control messages. The advantage of this type of framework is it isolats the high level decision-making functions such as ANS from the underlying technology dependent information as described in Section VI. It facilitates the introduction of new ANS mechanism without modifying other elements of the network. Finally, it is worth noting that this proposed solution is complementary to any network-controlled mobility management solution. It provides the end users with some level of control on the handoff decision. The diversity and the heterogeneity of available wireless access networks make the process very complex but at the same time create new opportunities to provide better services to end users.

\section{Network criteria and user preferences}

As previously mentioned, during its operation the MT collects information about surrounding available access networks and maintains a database of information that is necessary for decision-making regarding the selection of the best access network. The considered criteria takes into account both user preferences and network status. In the following, we present the most important criteria that will be considered in the ANS mechanism:

- Link quality: measured in terms of RSS. This parameter evaluates the signal quality of the available network.

- Monetary cost: different networks may have different charging policies. Therefore, in some situations the usage cost of a network service might be a discriminating factor in the selection.

- Battery lifetime: power consumption becomes a critical issue especially if a mobile terminal's battery is low. In this case, it might be preferable to select an access network technology that minimizes the energy consumption.

- MT velocity: this criterion is important to evaluate the probability that the MT remains within the network coverage in the near future. Including this parameter in the ANS selection means finding the best tradeoff between the quality of service in the current time and the risk of disconnection in the near future. For example, a terminal moving at a high speed might be discouraged from connecting to small cell-size networks to avoid getting quickly outside their range.

- Network load: this parameter, which measures the network congestion, needs to be considered in the ANS mechanism since a link quality apparently good in terms of RSS, might becomes very weak if the network is overloaded. Note that the network load parameter can not be measured from the terminal side without being connected to the target network. Therefore, this information should be periodically transmitted to the MTs.

For each of the above mentioned criteria, end users are able to express their preferences in terms of level of importance. A user preference indicates how important a criterion should be considered in the selection process compared to other ones. The preference weight for each criterion may differ depending on the application (i.e. streaming application vs. web navigator) or on the user localization (i.e. at home vs. in the office) [25]. It is envisioned that predefined values for the criteria will be grouped in a portfolio of profiles (e.g., cost minimizing profile, energy saving profile, etc.) that can be selected by the end user in his MT. A proper GUI (Generic User Interface) could facilitate the introduction of these preferences and profiles for the end user but the specification of such an interface is beyond the scope of this work.

In the following, we highlight how user preferences should be taken into account in ANS mechanisms. We first present how an appropriate utility function should capture the user sensitivity to network parameters. Then, we show how a multicriteria utility function should take into account all these parameters in the ANS decision-making.

\section{Novel User Single Criterion Utility Function}

In this section, we propose a new single criterion utility function to capture the user preferences. First, we provide the 
conditions which are notoriously required for a single criterion utility function. Then, we present the existing functions that satisfy these conditions and we identify which additional requirements need to be imposed to a single criterion utility as part of the multi-criteria optimization in the context of ANS. Finally, taking into account these conditions, we propose a new single criterion utility function. It is worth noting that our contribution in this section is twofold: i) we formalize the properties that an utility function must meet; ii) we propose a candidate function that fulfills all these conditions.

\section{A. New single-criterion utility requirements}

For sake of brevity, we remind the reader to [26] and [27] for a complete state-of-the-art on single criterion utility functions and their main characteristics. Here, we provide the key concepts as well as the identified properties which are required in the area of network selection.

Proper utility functions for network selection should exhibit the following properties:

1) twice differentiability,

2) monoton function,

3) concavity,

4) convexity.

It is well known that, if a given function $u(x)$ is suitable for an upward ${ }^{1}$ criterion utility, then $(1-u(x))$ is suitable for a downward criterion utility. We have therefore examined the utility forms for an upward criterion, assuming that the utility for downward criterion will be exactly the opposite one.

Among the numerous existing functions, we found that the ones that satisfy these necessary conditions are the sigmoidal (S-shaped) functions [11], [13], [28]

$$
\begin{array}{ll}
u_{1}(x)=\frac{1}{1+e^{\zeta\left(x_{m}-x\right)}} \quad\left(\zeta, x_{m}>0\right) \\
u_{2}(x)=\frac{\left(x / x_{m}\right)^{\zeta}}{1+\left(x / x_{m}\right)^{\zeta}} \quad\left(x_{m}>0, \zeta \geq 2\right)
\end{array}
$$

An interesting characteristic of these functions is that by tuning the parameter $\zeta$ the utility function shape changes, and therefore could capture more accurately the sensitivity of the utility to the variation of the criteria. This important key property is used here to derive the proposed overall user utility function. In particular, from the sigmoid functions $u_{1}(x)$ and $u_{2}(x)$ in (1) and (2), it holds that $u_{1}\left(x_{m}\right)=u_{2}\left(x_{m}\right)=0.5$. Value $x_{m}$ corresponds to the threshold between the satisfied and unsatisfied zones of a specific parameter. The values of $x_{m}$ and $\zeta$ determine the center and the steepness of the utility curve, respectively. Hence $\zeta$ allows the function to capture the user sensitivity to variation in access network characteristics as previously highlighted.

From the previous study, it appears that the sigmoidal form is suitable to model the user utility as a single criterion in the network selection process. However, it is quite challenging to tune the parameters of this function (i.e., $\zeta$ and $x_{m}$ ) to suit

\footnotetext{
${ }^{1}$ When the end-user QoE increases accordingly with given parameter (e.g., datarate), the parameter is an upward parameter/criterion. Similarly, a parameter that negatively affects the user satisfaction (e.g., cost) is a downward parameter/criterion.
}

the technological constraints as well as the user requirements (i.e., lower limit $x_{\alpha}$ and upper limit $x_{\beta}$ for each criterion) and sensitivity. For this reason, in addition to the four previous requirements, we introduce four additional conditions for the sigmoidal utility function:

$$
\begin{aligned}
u(x) & =0 \quad \forall x \leq x_{\alpha} \\
u(x) & =1 \quad \forall x \geq x_{\beta} \\
u\left(x_{m}\right) & =0.5 \text { for a given } x_{m}
\end{aligned}
$$

free steepness parameter

The motivation of adding these additional conditions is that each utility function should be able to take into account the lower limits (i.e., $x_{\alpha}$ ) and the upper limit (i.e., $x_{\beta}$ ), if exist. For example, the bandwidth allocated by an access network to a particular user needs to be bounded by its maximum system bandwidth. Regarding each considered criterion, the user should be able to say when he is fully satisfied (i.e., $u(x)=1$ ) and when he is definitively unsatisfied (i.e., $u(x)=0)$. This condition is not necessary when different alternative access networks are compared based on a single criterion. However, in the multi-criteria context, the same measure for all criteria should be considered. Since the criteria used in access network selection are not compensatory (i.e, a criterion is not totally compensated by another criterion or a set of other criteria) [26], the nullity and unity of utility become vital.

Furthermore, the utility function should retain a steepness parameter to model the user sensitivity to the criterion value change. The steepness is a free parameter that may be set differently for the different criteria. In particular, we use a sigmoid piecewise function: one convex piece for $x_{\alpha} \leq x \leq$ $x_{m}$ and one concave piece for $x_{m} \leq x \leq x_{\beta}{ }^{2}$. The two pieces should be designed to be continuous and differentiable at the point $x_{m}$. To achieve this, the two steepness parameters can be harmonized, knowing that each piece function has a free steepness parameter.

\section{B. Proposed Single-Criterion Utility Function}

To the best of our knowledge, an utility function able to meet all these eight highlighted requirements is still missing in the existing ANS mechanisms. Therefore, we propose a novel single-criterion utility function, modifying the sigmoid form (2) to take into account the identified additional requirements.

Proposition 4.1: Given a range of an upward criterion $x$, $x_{\alpha} \leq x \leq x_{\beta}<\infty$, and a middle point of the utility $x_{m}$, a suitable utility function for criterion $x$ is defined as:

$$
u(x)= \begin{cases}0 & x<x_{\alpha} \\ \frac{\left(\frac{x-x_{\alpha}}{x_{m}-x_{\alpha}}\right)^{\zeta}}{1+\left(\frac{x-x_{\alpha}}{x_{m}-x_{\alpha}}\right)^{\zeta}} & x_{\alpha} \leq x \leq x_{m} \\ 1-\frac{\left(\frac{x_{\beta}-x}{x_{\beta}-x_{m}}\right)^{\gamma}}{1+\left(\frac{x_{\beta}-x}{x_{\beta}-x_{m}}\right)^{\gamma}} & x_{m}<x \leq x_{\beta} \\ 1 & x>x_{\beta}\end{cases}
$$

${ }^{2}$ Note that $x_{m}$ is user-specific and not necessarily the median of the interval $\left[x_{\alpha}, x_{\beta}\right]$. 
where

$$
\begin{aligned}
\gamma & =\frac{\zeta\left(x_{\beta}-x_{m}\right)}{x_{m}-x_{\alpha}} \\
\zeta & \geq \max \left\{\frac{2\left(x_{m}-x_{\alpha}\right)}{x_{\beta}-x_{m}}, 2\right\}
\end{aligned}
$$

and $\zeta$ and $\gamma$ are the tuned steepness parameters.

Proof: We aim at showing that the proposed function satisfies the twice differentiability, concavity, convexity and also the conditions identified in (3)-(5). First, the proposed utility function clearly satisfies the conditions (3), (4) and (5). We see that (7b) and (7c) are similar to sigmoidal function $u_{2}(x)$. In order to show that (7b) and (7c) follow the increasing, concavity and convexity and twice differentiability conditions [13], we only need to show that $\zeta \geq 2, \gamma \geq 2$ and the first derivative of $u(x)$ is continuous at $x_{m}$. From (9), we have $\zeta \geq 2$ and $\zeta \geq \frac{2\left(x_{m}-x_{\alpha}\right)}{x_{\beta}-x_{m}}$. Substituting the latter to (8), we have $\gamma \geq 2$. As (7b) and (7c) are differentiable, we have:

$$
\begin{aligned}
\lim _{x \rightarrow x_{m}^{+}} u^{\prime}(x) & =\frac{\zeta}{4\left(x_{m}-x_{\alpha}\right)} \\
\lim _{x \rightarrow x_{m}^{-}} u^{\prime}(x) & =\frac{\gamma}{4\left(x_{\beta}-x_{m}\right)}
\end{aligned}
$$

By replacing (8) to (11), we have $\lim _{x \rightarrow x_{m}^{+}} u^{\prime}(x)=$ $\lim _{x \rightarrow x_{m}^{-}} u^{\prime}(x)$. Hence, $u(x)$ is twice differentiable therefore it satisfies all requirements of a utility function.

If a given criterion does not have an upper limit value (i.e., $x_{\beta}=\infty$ ), its utility will follow:

$$
u(x)= \begin{cases}\frac{\left(\frac{x-x_{\alpha}}{x_{m}-x_{\alpha}}\right)^{\zeta}}{1+\left(\frac{x-x_{\alpha}}{x_{m}-x_{\alpha}}\right)^{\zeta}} & x \geq x_{\alpha} \\ 0 & \text { otherwise }\end{cases}
$$

The form of the utility function for a downward criterion again is $(1-u(x))$ where $u(x)$ follows (17) or (12) depending on whether the upper limit value $x_{\beta}$ of the downward criterion exists or not. Our proposed utility form offers a practical way to model the expected user utility from a criterion with respect to given user- and technology-related parameters $x_{m}, x_{\alpha}$, and $x_{\beta}$. The steepness parameter $\zeta$ can therefore be tuned to capture more accurately the user preferences and sensitivity. In general, to model a high sensitivity of a user to the variation of a criterion, the value of $\zeta$ is set to a high value and vice versa.

\section{Novel Multi-Criteria Utility Function}

In the following, we show how several criteria can collectively impact the decision-making during network selection. Similarly to the previous section, we first identify and compare the existing multi-criteria utility functions that have been used so far in access network selection and radio resource management, highlighting the current limitations and the need for a novel aggregate utility function. Then, we introduce the requirements on the proper utility function, and we finally provide our multi-criteria aggregated utility function.
Table II

CASE STUDY: ADDITIVE MULTI-CRITERIA UTILITY

\begin{tabular}{|c|c|c|c|}
\hline Utility & $w_{i}$ & Network A & Network B \\
\hline$u($ Cost $)$ & $1 / 3$ & 0.5 & 0.8 \\
\hline$u(Q \circ S)$ & $1 / 3$ & 0.5 & 0.8 \\
\hline$u($ Load $)$ & $1 / 3$ & 0.5 & 0.05 \\
\hline Aggregate Utility & & $\mathbf{0 . 5}$ & $\mathbf{0 . 5 5}$ \\
\hline
\end{tabular}

\section{A. Existing Multi-Criteria Utility Functions}

The most common multi-criteria utility functions, that have been used so far in access network selection and radio resource management, are the additive aggregate utility and the acceptance probability. In the following, we provide the main limitations of these two approaches.

Additive aggregate utility. A common approach to compute the aggregate multi-criteria utility of an access network is to use the additive aggregation function given by [2]-[4], [29]

$$
U(\mathbf{x})=\sum_{i=1}^{n} w_{i} u_{i}\left(x_{i}\right), \quad \text { where } \sum_{i=1}^{n} w_{i}=1
$$

where $x=\left[x_{1}, \ldots, x_{n}\right]$ is the vector of $n$ considered criteria and $w_{i}$ are the user preference weights. Very similar to the scoring method, additive utility offers an easy and accessible approach to aggregate different elementary utilities, and it also allows users to introduce their preferences for different criteria.

However, the multi-criteria utility function in (13) is built on the assumption of independency between criteria. This assumption does not always lead to the best selection. An example of this limitation arises when an access network provides good utility for all selection criteria except for one, as shown in Table II. The access network A is the best one, since $\mathrm{B}$ is overloaded. However, the additive multi-criteria utility wrongly suggests to select the access network B since it has the highest aggregate utility. Obviously, the additive aggregate utility cannot prevent the MT users from selecting an access network for which an elementary utility is null i.e.,:

$$
\exists i \in\{1, . ., n\}: \lim _{u_{i}\left(x_{i}\right) \rightarrow 0} U(\mathbf{x}) \neq 0
$$

To conclude, we can state that a suitable aggregate utility should not vanish when the utility value of a single-criterion is close to zero.

Acceptance probability utility: The acceptance probability has been widely used in radio resource management to measure the probability that a user is satisfied with the perceived utility $u$ given the price $p$. Acceptance probability is expressed as follows

$$
A(u, p)=1-\exp \left(-C u^{\mu} p^{-\epsilon}\right)
$$

where $\mu>0$ and $\epsilon>0$ control the user sensitivity to utility and price, respectively, and $C$ is a positive constant representing the satisfaction reference value. This function is associated with the Cobb-Douglas demand curves [30].

The acceptance probability can overcome the limitations of the additive utility in (14), however, it still has three limitations when measuring the user's satisfaction: 
The first visible limitation is the zero price effect, or $\lim _{p \rightarrow 0} A(u, p)=1, \quad \forall u>0$. An access network whose usage price is close to zero (e.g., free public WiFi) will always be selected even if it offers the poorest quality. This is because the acceptance probability does not take into account foresighted penalties (i.e., service degradation in the future). One possible solution is to scale the price to interval $[0,1]$ with a downward utility function $u_{p}(p)$, i.e.,

$$
A(u, p)=1-\exp \left(-C u^{\mu} u_{p}(p)^{\epsilon}\right)
$$

The utility $u$ is computed for only one criterion (e.g., the allocated bandwidth). In multi-criteria network selection, the utility should include all characteristics except price $p$. The solution is then to define a global utility as either the product over a set of elementary utilities or the weighted average over elementary utilities as suggested in [31].

iii

Even if the overall utility is used and the zero price effect is solved, the resulting acceptance probability provides no means to take into account the user preference weights $w_{i}$ as in the case of the additive multi-criteria utility approach.

For all these reasons, it appears that the Cobb-Douglas acceptance probability is not appropriate to model aggregate utility or acceptance probability in the considered environment. In the following, we formalize these limitations providing the requirements that a suitable utility function should meet.

\section{B. New Multi-Criteria Requirements}

An important aspect to consider when designing the multicriteria utility function is how criteria affect each others during the aggregation process. It is important to ensure that the nullity of a specific elementary utility implies the elimination of the corresponding access network in the selection process. In general, when a non-zero preference weight is set for a criterion, it means that this criterion is important for the user in his quality of experience. Thus, if its utility is equal to zero (i.e., its value is below $x_{\alpha}$ for an upward criterion or above $x_{\beta}$ for a downward criterion), the corresponding access network does not satisfy the technical or user constraints. A rational decision should be to not select this network; however, the non-zero preference criteria are not independent from each other. This means that, unlike many other contributions, we believe that the aggregate utility function should reflect this interdependency. Therefore, we define the following design requirements for a new multi-criteria utility function:

$$
\begin{aligned}
\frac{\partial U(\mathbf{x})}{\partial u_{i}} & \geq 0 \\
\operatorname{sign}\left(\frac{\partial U(\mathbf{x})}{\partial x_{i}}\right) & =\operatorname{sign}\left(u_{i}^{\prime}\left(x_{i}\right)\right) \\
\lim _{u_{i} \rightarrow 0} U(\mathbf{x}) & =0 \quad \forall i=1 . . n \\
\lim _{u_{1}, . ., u_{n} \rightarrow 1} U(\mathbf{x}) & =1
\end{aligned}
$$

The aggregate utility should increase when the elementary utility increases, condition (17). It should be an increasing function of upward criteria and a decreasing function of downward criteria, condition (18). The condition (19) is indeed a weak condition to resolve the close-to-zero elementary utility effect observed in the additive aggregate utility. It eliminates the access networks having a zero elementary utility in the decision-making process. In addition, the aggregate utility should be able to downgrade the rank of the access network that has a close-to-zero elementary utility. The condition (20) reflects the fact that if all elementary utilities are equal to 1 (i.e., all criteria satisfy the user's expectation), the aggregate utility should be also equal to 1 . Finally, it is necessary to include the user preference weights of different criteria in the aggregate utility form to take into account the user preferences priority ranking.

\section{Proposed Multi-Criteria Utility Function}

In this section, we propose a new multi-criteria utility function and we proof that it meets the above mentioned requirements.

Proposition 5.1: Given a network selection criteria vector $\mathbf{x}$ and an associated preference vector $\mathbf{w}$, a suitable multi-criteria utility function is formulated as:

$$
U(\mathbf{x})=\prod_{i=1}^{n}\left[u_{i}\left(x_{i}\right)\right]^{w_{i}}
$$

where $n$ is the size of vector $\mathbf{x}, w_{i}$ is the preference weight for criterion $i\left(\sum_{i=1}^{n} w_{i}=1\right)$, and $u_{i}\left(x_{i}\right)$ is the elementary utility of criterion $i$ that follows the utility form proposed in Proposition 4.1.

Proof: We show that the proposed form satisfies the identified requirements. This multiplicative form reflects well the interdependence between the criteria and can eliminate the close-to-zero effect. It is easy to verify that the proposed multi-criteria utility satisfies the requirements (19) and (20). Moreover, (17) is verified as $U(\mathbf{x})$ is an increasing function of each $u_{j}$. Indeed,

$$
\frac{\partial U(\mathbf{x})}{\partial u_{j}}=w_{j}\left[u_{j}\left(x_{j}\right)\right]^{\left(w_{j}-1\right)} \prod_{i \neq j}^{n}\left[u_{i}\left(x_{i}\right)\right]^{w_{i}} \geq 0
$$

Also, the partial derivative of $U(\mathbf{x})$ at an $x_{j}$ is given as

$$
\begin{aligned}
\frac{\partial U(\mathbf{x})}{\partial x_{j}} & =\left(w_{j}\left[u_{j}\left(x_{j}\right)\right]^{\left(w_{j}-1\right)} \prod_{i \neq j}^{n}\left[u_{i}\left(x_{i}\right)\right]^{w_{i}}\right) u_{j}^{\prime}\left(x_{j}\right) \\
& =\frac{\partial U(\mathbf{x})}{\partial u_{j}} u_{j}^{\prime}\left(x_{j}\right)
\end{aligned}
$$

This proves that $U(\mathbf{x})$ is increasing for upward criteria and decreasing for downward criteria therefore (18) is verified.

Finally, we need to verify if $w_{i}$ could really represent the user preferences. As a monotonic transformation of a utility produces another utility with the same preference ranking, we can apply a logarithm transformation to $U(\mathbf{x})$ :

$$
V(\mathbf{x})=\ln (U(\mathbf{x}))=\sum_{i=1}^{n} w_{i} \ln \left(u_{i}\left(x_{i}\right)\right)
$$


If $v_{i}\left(x_{i}\right)=\ln \left(u_{i}\left(x_{i}\right)\right)$, we see that $v_{i}\left(x_{i}\right)$ is an elementary utility function of criterion $i$ (by the monotonic transformation property). We have

$$
V(\mathbf{x})=\sum_{i=1}^{n} w_{i} v_{i}\left(x_{i}\right) \sim U(\mathbf{x})
$$

Under this additive presentation, we clearly see that $w_{i}$ are the user preferences. So the equation(21) is a suitable multicriteria utility form.

The proposed multi-criteria utility satisfies all requirements of an aggregate utility function and overcomes the limitations of existing models. Along with the limitations of the acceptance probability identified in Section V-A, a suitable acceptance probability model should follow (24), (25) and (26).

$$
\frac{\partial A(\mathbf{x})}{\partial x_{i}} \geq 0 \quad \frac{\partial A(\mathbf{x})}{\partial x_{j}} \leq 0
$$

where $x_{i}$ is an upward criterion and $x_{j}$ is a downward criterion and to model how the utility changes with the changes of these two types of criteria.

$$
\forall x_{i} \in \mathbf{x}: u_{i}\left(x_{i}\right)=1 \Rightarrow A(\mathbf{x})=1
$$

This is to ensure that when all criteria satisfy the user's preferences (i.e., the utility of very criterion is equal to 1), the user has no rational reason to refuse the service.

$$
\exists x_{i} \in \mathbf{x}: u_{i}\left(x_{i}\right)=0 \Rightarrow A(\mathbf{x}, \mathbf{w})=0
$$

This to verify that when the utility of any criteria is vanished, the user should not accept the service.

It can be seen that the proposed weighted multiplicative utility form in Proposition 5.1 can be eventually used to properly model the user acceptance probability. Hence, we have:

$$
A(\mathbf{x}, \mathbf{w})=\prod_{i=1}^{n}\left[u_{i}\left(x_{i}\right)\right]^{w_{i}}
$$

As demonstrated in the proof of Proposition 5.1, this weighted multiplicative aggregate form fully satisfies the conditions (24), (25) and (26).

\section{MOBILITY MANAGEMENT AND INTEGRATION SOLUTION FOR HETEROGENEOUS NETWORKS}

The following section shows how the proposed utility functions are used in the ANS module and integrated in MT operating system. In particular, details on how the handover process is handled across heterogeneous networks are provided, describing the MIH framework and its benefit in the ANS mechanisms.

MIH framework defined by the standardisation group IEEE 802.21 is intended to facilitate handover and interoperability between IEEE 802 and non- IEEE 802 technologies (e.g., UMTS, LTE) in order to provide transparent service continuity across heterogeneous networks. The MIH consists of a signaling framework and triggers that make information available from lower layers (MAC and PHY) to higher layers of the protocol stack (network to application layers). MIH is responsible for unifying the various Layer 2 (L2) technology

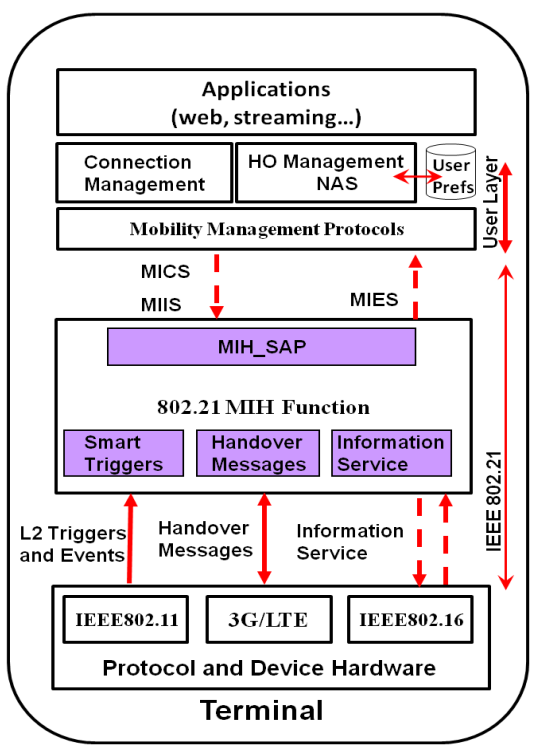

Figure 2. MIH Framework at the Terminal Side.

information used by the handover decision algorithms so that the upper layers can abstract the heterogeneity aspects inherent to various underlying heterogeneous technologies. The lifecycle of handover procedure is composed of three consecutive phases that are: handover initiation, handover preparation and handover execution. During the handover initiation, the MT searches for new links and performs three main tasks network discovery, network selection and handover negotiation. Handover initiation can be triggered by different factors such as: monetary cost, link quality, and in our case predefined user preferences. In the second phase, the MT sets up the new link and performs the mobility at the IP level. In the final phase (the handover execution) the MT achieves the transfer of the session and starts to send/receive packets using the selected network.

Our ANS mechanism addresses the network selection part of the process involving the complete handover. MIH framework helps to manage the two first phases of the handover life cycle maintaining a database of information about all available networks and hiding the heterogeneity of underlying data on which these information are based. Fig. 2 depicts the main component of the MIH framework. The IEEE 802.21 local or remote L2 interfaces deliver events and triggers to the MIH function (MIHF) layer called link events. These MIH events are made available to upper layers through the MIH SAP (Service Access Point). The handover management module that includes the ANS mechanism uses these MIH events to discover available networks and to select the best access network. Some important events are listed in the following with their meaning:

1) MIH Link Up: L2 connection is established and link is available for use.

2) MIH Link Going Down: Link conditions are degrading and connection loss is imminent.

3) MIH Link Detected: A new link has been detected.

The MIH framework introduces a Handover Policy Control 
Function component located at the user layer of the MIH framework. It is implemented as an agent and uses the services provided by the MIHF through its MIH SAP in order to optimize the handover process. This agent could control in a homogeneous manner any underlying physical, data link or logical link layers. It can receive events and/or messages from any underlying interface to indicate changes in their state and information about their behavior. The proposed ANS mechanism is implemented in this agent. The end user preferences regarding selected criteria (e.g., bitrate, cost, power consumption, etc.) are collected and stored in the MT in the user preference database.

\section{Multi-Technologies Simulator}

In order to evaluate our solution against other approaches, we have implemented an appropriate simulation environment [32]. The simulation engine that have been used is NS-2 [33] version 2.29 with NIST add-on modules [34]. In the following, we describe the most important modules that have been added in order to support the realistic mobility scenarios described in the previous section.

\section{A. Additional IEEE 802.21 add-on module}

The 802.21 add-on module is an implementation of MIHF based on draft 3 of IEEE 802.21 specification. The most important MIH event that is considered in this simulation is the MIH Link Going Down Event [34]. This event is triggered when the power level between two consecutive packets at the receiver is decreasing or when the power level of a packet is lower than the threshold signal strength of one received packet (i.e., RxThresh). In order to identify the power level to trigger the MIH Link Going Down Event in the simulation and to accordingly update the utility of each "going down" interface, we have defined a new event called MIH Multi Utility Event. If one frame is received at the MAC layer with a signal strength higher than RxThresh, it is set as valid for decoding otherwise it is tagged as corrupted and the frame is discarded. This MIH event informs the MT of an imminent handover and therefore the need for selecting another better available access network.

\section{B. Enhanced Neighbor Discovery (ND) add-on module}

ND module was designed to provide movement detection at layer 3. Its role is to manage the IP addresses when the access network is changed. The module is a part of MIH framework and can support multiple interface types such as UMTS, WiFi and WiMAX. The corresponding ND agent is included in all nodes and its behavior changes depending on whether the node is a router or a host. If it is a router, the embedded Router ND agent periodically sends Router Advertisement (RA) messages to the hosts and receives Router Solicitation (RS) messages. If it is a host, the existing embedded Host ND Agent sends RS messages to contact a Router ND agent in the network to solicit a route and the Router ND Agent replies with RA message. The received prefix information in the RA is compared to the existing values and possibly new values are added.

\section{Virtual Nodes with Multiple Interfaces}

Supporting nodes with multiple interfaces is not intuitive in NS-2. This is because external packages do not necessarily follow the same node structure as the one defined in the basic model. To resolve this issue, NIST created the concept of MultiFace Node (MFN), which is a virtual node that links to other nodes and integrates the ND and MIH modules. The other nodes are considered as interfaces for the multiFace node. MFN aims to simplify the management of heterogeneous interfaces to implement efficient access network selection algorithms. However, the VHO algorithm implemented by the NIST in the version 2.29 of NS2 only considers the RSS for the decision-making. We have modified this node to support our proposed ANS mechanism.

We have extended all the modules such that all heterogeneous interfaces of the MT can be collected. In addition, it is possible now to control their behavior using MIH commands as recommended by the IEEE 802.21 standard. Finally and importantly, we have added the possibility to use the LTE technology as detailed in the following subsection.

\section{Integration of LTE-Sim with NS2-29}

Currently the NIST add-on modules in NS-2 only support WiMAX, Wi-Fi, UMTS and Ethernet technologies. In order to evaluate our ANS solution in the context of existing and future technologies, we have included in our simulation platform the LTE-Sim simulator [35]. The LTE-Sim is an open source framework that provides a complete performance verification of the LTE system. It encompasses several aspects of LTE networks including the Evolved Universal Terrestrial Radio Access (E-UTRAN) and the Evolved Packet System (EPS). Particularly, it supports multi-users single and multi-cell environments, QoS and flow management, user mobility and handover procedures. The LTE simulator implements three kinds of network nodes: User Equipment (UE), Evolved nodeB (eNB) and Mobility Management Entity/Gateway (MME/GW). Each of these node can be a source or a destination of data flows. This simulator manages the physical layers of both MTs and eNBs and supports radio resource management. Both downlink and uplink scheduling strategies are defined in a MAC entity. Moreover, LTE-Sim is able to simulate various traffic generators: Video-trace based, VoIP G.729, CBR and infinite buffer based on an ideal greedy source. To simulate our environment we have loosely integrated the two simulators in a system where both platforms are running in parallel (i.e. the NS-2 scheduler stops while the LTE-Sim is running during a period of time and eventually sends performance reports). The mobility model is negotiated in the first part of the simulation and is shared between the two platforms via the gateway module. This allows LTE-Sim to know the position of each MT at any time and to execute the mobility scenario. We have also created in NS-2 a new type of node which represents the LTE interface and integrated it in the Virtual MultiFace Node. After each time period $\delta_{L T E}$, the MT computes the utility of the LTE interface based on the preferences of the user and the network parameters. If the LTE interface utility is lower than the maximum of the 3 others interfaces (WiFi, WiMaX and 
UMTS), the MT does not handover to the LTE network and maintains its connectivity in NS-2. On the other hand, if the utility of the LTE interface is the highest one then the MT triggers a VHO during a period $\delta_{L T E}$ and handover to the LTE access network. In the LTE-Sim a Horizontal Handover (HHO) algorithm is supported, and if an HHO occurs, an event is created in the NS-2 simulator to let it informed about the situation of the MT within the LTE network.

\section{E. Network coverage and mobility model}

To simulate a realistic mobility scenario, we have used the well known mobility emulator CanuMobiSim [36] to compel the movement of MTs and generates user's movements trace file written in TCL [37] and used them in the simulation platform. We have created several multi-interfaces MTs that move within a defined area following the generated movements trace file. During their move, MTs are always within the range of one or several wireless access networks (i.e., WiFi, WiMAX, UMTS and LTE) and are also able to detect them.

\section{EVAluation of The END Users BenEFit}

We now provide results showing the benefit that the end user can achieve from our proposed ANS solution with respect to the baseline additive utility function. To compute the baseline utilities, we have considered the original sigmoid form in (2), while the sigmoid form in Proposition 4.1 has been used to compute the elementary utilities in our proposed multiplicative multi-criteria utility. The considered network topology is composed of one WiMAX base station (BS), four WiFi APs, one 3G/UMTS and 7 LTE eNodeB cells positioned in the environment so that partial or complete overlapping between different technologies exists. Several MTs (up to 20) move around within the area and are covered by one or several networks.

At time $t=0$, the MTs are randomly distributed in the area and are connected by default to the $3 \mathrm{G}$ cell. At each periodic time unit, the MT moves within the area. During this movement, the MT scans available networks, calculates their utility and decides if it is better to remain connected to the current network or if it is necessary to handoff to a better one. Table I presents the values for the most relevant parameters of the access networks.

\section{A. Simulations scenarios and parameters}

To evaluate the benefit for the end users, we have defined a scenario where a user, equipped with an ANS enabled terminal, has a preference for streaming application, characterized by a specific traffic profile (i.e, constant bit rate). In this scenario and type of service, the user sets his preferences prioritizing the data rate vs the price. In order to compare the two approaches (additive and multiplicative aggregate utility function), the playback buffer level in the MT is monitored. The buffer level indicates the playout duration for which media data is available. The video stream is displayed to the user as far as data is available in the buffer. If the buffer runs empty, the service is interrupted (i.e., rebuffering phase), leading to
Table III

PARAMETERS FOR UTILITY COMPUTATION

\begin{tabular}{|c|c|c|c|c|c|}
\hline Criterion & Preference & $x_{m}$ & $x_{\alpha}$ & $x_{\beta}$ & $\zeta$ \\
\hline Bandwidth $(b)$ & $w_{1}=0.5$ & 40 & 5 & 90 & 2 \\
\hline Price $(p)$ & $w_{2}=0.5$ & 30 & 0 & 80 & 3 \\
\hline
\end{tabular}

a bad experience for the user. Note that the rebuffering phase has been identified as one of the dominating QoE impairment [38].

At $t=0$, the buffer is assumed to be fully filled. At every time unit, the streaming application retrieves media data from the buffer at a rate $R_{\text {play }}$ to play the video stream on the MT screen. At the same time, the buffer is refilled with media data coming from the network at the current throughput rate $t p$. This process is specified in the following formulation of the buffer size calculation:

$\left\{\begin{array}{l}\text { buf }[0]=3 R_{\text {play }} \\ \text { buf }[t]=\max \left(0, \min \left(3 R_{\text {play }},\left(\text { buf }[t-1]+t p[t]-R_{\text {play }}\right)\right)\right)\end{array}\right.$

where $R_{\text {play }}$ is the playback rate of the streaming application and $3 R_{\text {play }}$ is the maximal memory size allocated to the buffer.

1) Parameters for numerical analysis: For numerical analysis, we assume a basic scenario where the ANS decision is based on only two criteria that are the allocated bandwidth $b$ and the price $p$ given in Table III.

2) Parameters for the network simulator stacks: In order to evaluate the effect of the communication stacks parameters on the ANS decisions, we have enlarged the number of criteria to five: cost $(c)$, power consumption gains ( $g e$ ), maximum achievable data rate $(r)$, network load $(\rho)$ and Frame Error Rate (FER). We consider in this simulation the cost per downloaded data volume (cents/Kbit) for streaming and data downloading applications. The power consumption gain parameter is defined as the ratio between the power consumption rate $e_{i}$ and the maximum achievable data rate $r_{i}$, i.e.,

$$
g e_{i}=\frac{e_{i}}{r_{i}}(J / M b) .
$$

The energy consumption rate values of UMTS and WiFi interfaces are the averaged values taken from the range presented in [39] as well as in the off-the-shelf products specification. In general, the energy consumption rate of WiMAX interface is greater than the UMTS/LTE interface and not much less than the WiFi interface [24]. The FER of an access network can be estimated based on the link quality and the corresponding modulation and coding scheme. The value range of selection criteria are given in Table IV, while the setting parameters for each elementary utility used in the simulation are given in Table V.

To detect any IEEE 802.21 state change event (e.g., Link Detected, Link UP, Link Going Down, Link Down), these parameters are evaluated at a periodic time in the MAC layer of each MT. The technical characteristics of the access networks using the WiFi, UMTS, LTE and WiMAX technologies are depicted in Table I. 
Table IV

SIMULATION PARAMETERS FOR USE CASES

\begin{tabular}{|c|l|l|l|l|}
\hline & UMTS R99 & LTE & WiFi & WiMAX \\
\hline cost(cents/Kb) & $20-60$ & $20-50$ & $1-40$ & $1-50$ \\
\hline $\mathrm{e}(\mathrm{W})$ in active state & 1.2 & 2.5 & 4.5 & 3.5 \\
\hline Data rate $\mathrm{r}(\mathrm{Kbps})$ & $100-1500$ & $300-1800$ & $100-1500$ & $100-1800$ \\
\hline Network load $\rho$ & $0-0.5$ & $0-0.5$ & $0-0.5$ & $0-0.5$ \\
\hline FER & $0.0005-0.08$ & $0.0005-0.08$ & $0.0005-0.08$ & $0.0005-0.08$ \\
\hline
\end{tabular}

Table V

PARAMETERS SETTINGS FOR ELEMENTARY UTILITY FORMS

\begin{tabular}{|c|c|c|c|c|}
\hline Criterion & $x_{\alpha}$ & $x_{\beta}$ & $x_{m}$ & $\zeta$ \\
\hline$c($ cents $/ \mathrm{Kb})$ & 5 & 70 & 35 & 2 \\
\hline$g e(J / M b)$ & 1 & 50 & 15 & 2 \\
\hline$r(K b p s)$ & 10 & 3000 & 800 & 3 \\
\hline$\rho$ & 0 & 1 & 0.5 & 2 \\
\hline$F E R$ & 0 & 0.2 & 0.1 & 3 \\
\hline
\end{tabular}
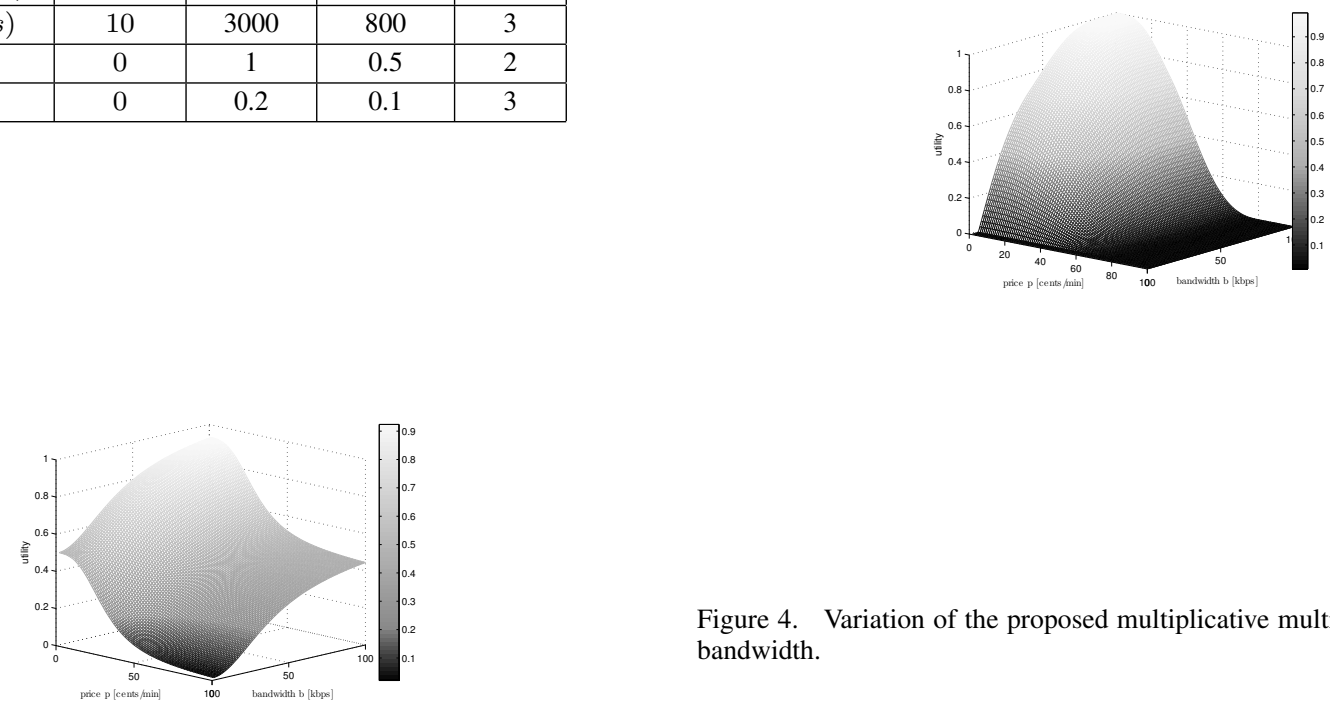

Figure 3. Variation of the additive multi-criteria utility vs price and bandwidth.

\section{B. Results}

To validate our proposal, we carried out two simulation campaigns. The first one, using matlab, aims at validating the proposed multiplicative utility function and at highlighting its superiority against the additive one. This is addressed from both the end-users side and also from the operator side. The second simulation campaign uses the built multitechnology simulation platform previously described including the implementation of the proposed ANS mechanism in MTs.

1) Results obtained with numerical analysis: In this first scenario, the obtained utility values of the additive multicriteria utility function and the proposed weighted multiplicative multi-criteria utility function are plotted in Fig. 3 and in Fig. 4 as function of both the price and the bandwidth. The shape of the additive multi-criteria utility form presented in

Figure 4. Variation of the proposed multiplicative multi-criteria vs price and bandwidth.

Fig. 3 highlights the limitations of the general additive models previously discussed. For example, Fig. 3 shows that an access network with $(p=1, b=1)$ has a higher utility than an access network with $(p=30, b=40)$ (denoted as $(p=1, b=$ $1) \succ(p=30, b=40))$. This leads to a bad decision for the user since the access network with $(p=1, b=1)$ could not satisfy the user's bandwidth requirements (minimum $b_{\alpha}=5$ as shown in the Table III). On the contrary the access network with $(p=30, b=40)$ fully meets the user expectations in both bandwidth and price but is not selected as the access. Similarly, $(p=100, b=100) \succ(p=80, b=90)$ is also not justified since the network of $(100,100)$ does not satisfy the user's constraint on the price (i.e., $p_{\beta}=80$ as specified in Table III). While the network of $(p=80, b=90)$ meets both bandwidth and price constraints. One may conclude that the identified limitations are due to the fact that original singlecriterion utility does not capture the limits of each criterion. To verify this, we replaced the original single-criterion utilities by the new proposed ones in the additive aggregate utility. The resulting form of the utility variation turns out to be very similar to the one shown in Fig. 3 but the obtained maximum aggregate utility is now equal to 1 .

However, all these limitations are overcome by our proposed utility form, as shown in Fig. 4. The proposed multiplicative form for ANS reflects correctly the interdependence among 


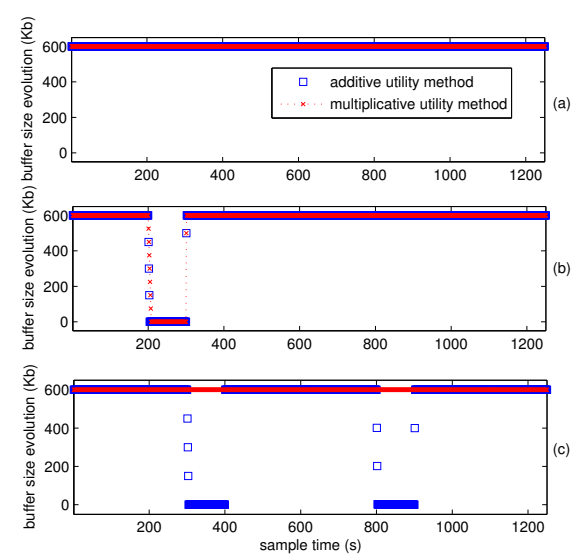

Figure 5. MT buffer size variation and multimedia service interruption comparison between additive utility based ANS and multiplicative utility ANS.

the criteria, avoiding the close-to-zero elementary utility effect which confirms the suitability of our single-criterion and multicriteria utility forms in efficiently capturing users' preferences.

At each simulation time, the access network selection is triggered and the buffers are updated. The simulation results of the three different network realizations are depicted in Fig. 5. In the first scenario presented in Fig. 5(a), both schema perform ideally. In the second scenario presented in Fig. 5(b), there is an outage episode. This is due to the fact that none available access networks satisfies the user's application requirements independently of the ANS schema. Finally, Fig. 5(c) shows a case in which the additive utility-based ANS schema leads to two rebuffering phases, because the schema was not capable of enforcing the best network selection while our weighted multiplicative utility-based schema allows the service to be delivered seamlessly during the whole simulation. This can be explained by the limitations of the additive approach to deal correctly with low cost weak access networks.

With the additive solution, the interruption time represents about $7 \%$ of all running time, whereas our multiplicative solution shows a total interruption ratio of less than $1 \%$ of the total time. This confirms that our solution outperforms the additive solution and behaves as required.

2) Results obtained with the simulation platform: The objective of the second simulation activity is to simulate the same scenario of streaming and data downloading applications using the simulation platform described in VII.

We have considered four single metric criteria

1) Static selection strategy: consists on statically ranking different access networks and to select the access network in a decreasing order of availability.

2) Cost minimization strategy: consists on assigning the highest priority to the cheapest access network.

3) Energy saving strategy: consists on assigning the highest priority to the technologies that consume less energy.

4) SNR maximization strategy: consists on assigning the highest priority to the network that has the highest Signal to Noise Ratio at the MT.

Each single-criterion strategy is compared to the proposed multi-criteria strategy and performance in terms of both of

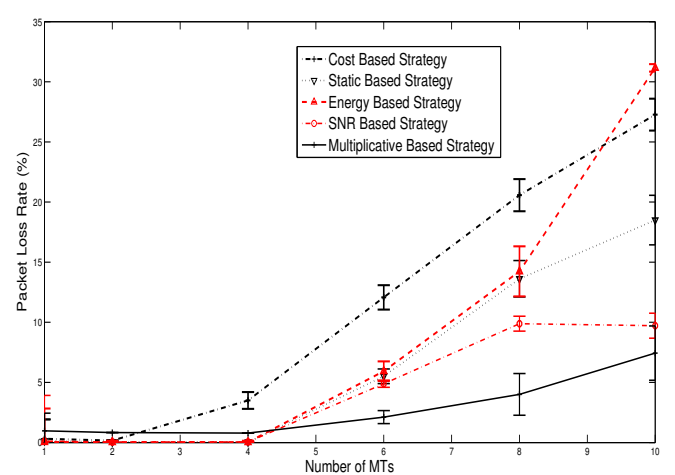

Figure 6. Comparison between different ANS strategies: packet loss rate versus number of MTs.

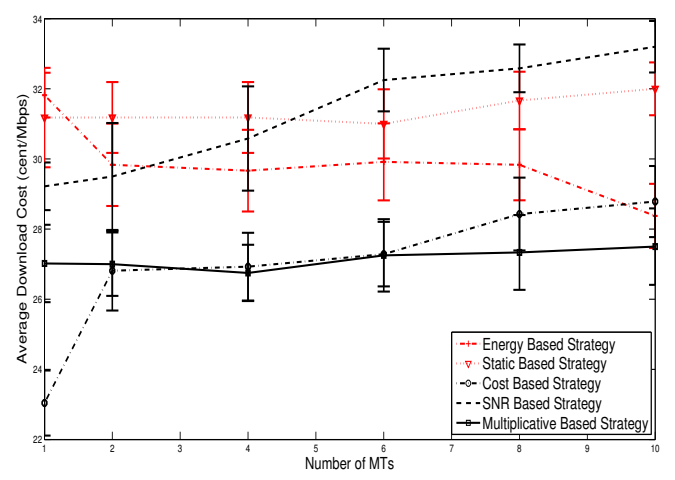

Figure 7. Averaged packet cost per downloaded data volume vs number of MTs.

packet loss rate (PLR) (Fig. 6) and average cost (Fig. 7). Results show that the multi-criteria based selection outperforms all other strategies. The reason is that multi-criteria utility function is constantly selecting the access network able to find the best balance among all criteria.

\section{Evaluation of the Network Operator Benefit}

Showed that the proposed ANS solution improves the benefit of the end-users in a context of multiple wireless access networks, it is important to see how this new ANS mechanism impact the network operators business, i.e., their income. For that, the network operator revenue, achieved when our multiplicative utility function is used, is compared to the revenue of the operator when the widely used Cobb-Douglas acceptance probability [13], [30] is adopted. The acceptance function is given by (15), with $C=2, \mu=2$ and $\epsilon=0.2$, such that the value of $A$ is always in the interval $[0,1]$ and it can be used as an acceptance probability.

\section{A. Parameters of the scenario}

Similarly to other contributions, we consider a scenario where a wireless network is deployed by an operator and $N_{\max }$ 
Table VI

Simulation PARAMETERS: OPERATOR CASE

\begin{tabular}{|c|c|c|c|c|c|c|c|}
\hline Class & Price & $\mathbf{w}=\left(w_{p}, w_{b}\right)$ & $p_{m}$ & $b_{m}$ & $\mu$ & $\epsilon$ & $\phi$ \\
\hline Silver & $p_{s}=20$ & $w_{s}=(0.6,0.4)$ & 20 & 30 & 2 & 6 & 10 \\
\hline Gold & $p_{g}=40$ & $w_{g}=(0.3,0.7)$ & 40 & 60 & 3 & 4 & 40 \\
\hline
\end{tabular}

maximum users can be simultaneously served, [13], [30]. We define two classes of service and suppose that users subscribe to only one of these services, i.e., $N_{s}$ silver users and $N_{g}$ gold users, with $N_{g}+N_{s}=N_{\max }$. The gold service ensures that the subscribers of this service will be better served than any subscriber of the silver service and obviously the pricing for this service is higher. The ANS algorithm is here based on both the price and the allocated bandwidth per user.

The total bandwidth available at a particular access network $C_{B W}$ depends on its technology (WiFi, UMTS, LTE or WiMAX). We consider the same network topology and radio resource allocation scenario as previously defined. We also consider MTs equipped with multiple wireless interfaces capable to connect to any of these wireless access networks.

The business objective of the operator is to maximize its revenue, i.e., the Expected Revenue (ER), while trying to satisfy as much as possible all its customers' preferences. To reach this aim, first the network operator estimates the acceptance probability of each user based on the maximum number of users that can be served and the total available bandwidth. Then, the efficient allocation of resources (i.e., the maximum bandwidth for each user depending on its contract) is defined. Once this efficient allocation of resources is identified, the result can be used by the network call acceptance control to accept or reject end users connection requests. In particular, the operator implements a strategy that maximizes its revenue given the available resources, accepting the user connections if the requested bandwidth does not exceed the the identified one.

For the sake of comparison, the ER is expressed as a function of the users acceptance probability using both the Cobb-Douglas and our proposed multiplicative utility function. The ER for the operator is estimated as follows:

$$
E R\left(\mathbf{b}, p_{s}, p_{g}\right)=\sum_{k=1}^{N_{s}} p_{s} A_{k}\left(b_{k}, p_{s}\right)+\sum_{k=N_{s}+1}^{N_{\max }} p_{g} A_{k}\left(b_{k}, p_{g}\right)
$$

where $p_{s}$ and $p_{g}$ are the flat prices paid by all users in the same class of service (i.e. silver and gold), $b_{k}$ is the bandwidth dedicated to the user $k$, and $\mathbf{b}=\left[b_{1}, \ldots, b_{N_{\max }}\right]$.

For the Cobb-Douglas case, $A_{k}$ is the Cobb-Douglas based utility acceptance probability of user $k$ given as a function of the available bandwidth $b_{k}$ and the price $p_{s}$ or $p_{g}$ :

$$
A_{k}\left(b_{k}, p_{k}\right)=1-e^{-K\left[u_{b_{k}}\left(b_{k}\right)\right]^{\mu}\left(p_{k} / \phi\right)^{-\epsilon}}
$$

where $K=-\log (0.9)$, and the other parameters are detailed in Table VI. Note that in (30) we have also imposed that

$$
p_{k}= \begin{cases}p_{s} & \text { if } 1 \leq k \leq N_{s} \\ p_{g} & \text { if } N_{s}<k \leq N_{\max }\end{cases}
$$

When the multiplicative utility function proposed in (27) is considered, the users preferences are taken into account in the acceptance probability estimation as follows

$$
A_{k}\left(b_{k}, p_{k}\right)=\left[u_{b_{k}}\left(b_{k}\right)\right]^{w_{b}}\left[u_{p_{k}}\left(p_{k}\right)\right]^{w_{p}}
$$

We assume that the operator has good information about its subscribers' preferences $\left(w_{s}\right.$ and $\left.w_{g}\right)$ as well as their sensitivities to quality of service (i.e. bandwidth in this case) and price $(\mu$ and $\epsilon)$ in the Cobb-Douglas case. The parameters for each class of service are presented in Table VI. The values $p_{m}$ and $b_{m}$ are the same values $x_{m}$ used in the single-criterion utility for price and bandwidth, respectively. The upper and lower limits of each criterion are set as $\left(p_{\alpha}=0, p_{\beta}=50\right)$ and $\left(b_{\alpha}=0, b_{\beta}=100\right)$.

To achieve a fair comparison between the Cobb-Douglas and our multiplicative acceptance probability (since they do not calculate the revenue in the same way), we introduce a new metric named Resource Efficiency Index (REI), which is defined as the ratio between the operator revenue and the potentially allocated resource. The REI is calculate as follows:

$$
\begin{aligned}
R E I\left(\mathbf{b}, p_{s}, p_{g}\right) & =\frac{R\left(p_{s}, p_{g}\right)}{B\left(\mathbf{b}, p_{s}, p_{g}\right)} \\
\text { where } B\left(\mathbf{b}, p_{s}, p_{g}\right) & =\sum_{k=1}^{N_{\max }} b_{k} A_{k}\left(b_{k}, p_{k}\right) \\
R\left(p_{s}, p_{g}\right) & =\sum_{k=1}^{N_{s}} p_{s} \theta_{s}(k)+\sum_{k=N_{s}+1}^{N_{\max }} p_{g} \theta_{g}(k)
\end{aligned}
$$

where $\theta_{g}(k)=1$ if the $k$ th user is a gold one, $\theta_{g}(k)=0$ otherwise, and $\theta_{s}(k)=1$ if the $k$ th user is a silver one, $\theta_{s}(k)=0$ otherwise. The REI represents the amount of expected income per unit for the allocated resource.

At each stage of the simulations, the objective is to find the most appropriate resource distribution vector $\mathbf{b}^{\star}=$ $\left[b_{1}^{\star}, \ldots, b_{N_{\max }}^{\star}\right]$ that maximizes the operator $R E I$, subject to available bandwidth $C_{B W}$. Once this efficient allocation vector calculated, the next step is the management of the connection requests (i.e. acceptance or rejection of the connections) in such a way that the operator reward is maximized and the bandwidth per user does not exceed the value identified in the vector $\mathbf{b}^{\star}$.

The simulation is conducted in two phases that are:

1) Initial efficient resource allocation (off-line phase).

2) Connections management and revenue calculation (online phase).

Phase 1: Efficient resources allocation evaluation. The first phase is executed off-line and is aimed at calculating the best resource allocation vector $\mathbf{b}^{\star}$. The input is the total number of subscribers as well as their service profile (gold or silver). Let $N_{g}$ and $N_{s}$ be the respective number of end users subscribing for a gold and silver contracts, and let suppose the network capacity in term of bandwidth $C_{B W}$ and the users' preferences are known. The proposed Algorithm 1 aims to compute the best allocation vector that maximizes $R E\left(\mathbf{b}^{\star}, p_{s}, p_{g}\right)$.

To find the best $\mathbf{b}^{\star}$, the algorithm relies on a heuristic approach which consists on a random walk in the search space 


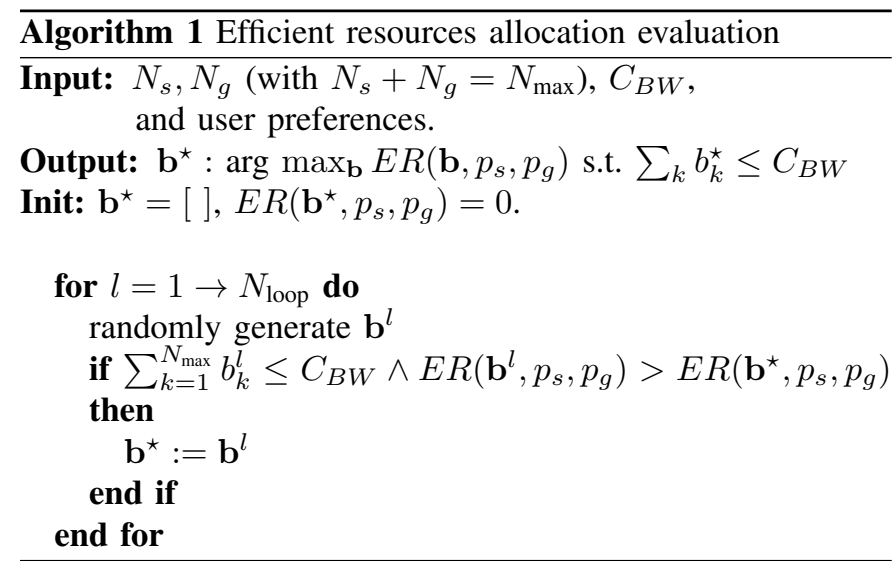

of acceptable resource distribution vectors $\mathbf{b}$. The algorithm improves at each stage the allocation vector similarly to a Monte Carlo method. The number of evaluation loops that are required to reach an optimal value for the vector depends on both the dimension of the search space and the walking step. In our case, after numerous tests, we have set this value $N_{\text {loop }}$ to $10^{6}$.

Phase 2: Users connections management and rewards evaluation. We now describe the second step of the simulations done in real-time. At each connection request initiated by a MT to any access network (WiMAX/WiFi/UMTS/LTE), the information contained in the message (e.g., Node ID, Requested Bandwidth, etc.) is extracted by the corresponding access network and added to a set of vectors called Resources Request Vectors Req. Among all the information provided, we denote by $\mathbf{b}^{\text {(Req) }}$ the requested bandwidth. Note that $\mathbf{b}_{k}^{(\mathrm{Req})}=\left[b_{1}^{(\mathrm{Req})} \ldots b_{N_{\max }}^{(\mathrm{Req})}\right]$, where $b_{k}^{\text {(Req) }}=0$ if the $k$ th user does not make any service request.

Every $\Delta t$ seconds, the network executes a connection acceptance control. In particular, the network processes the MTs requests and decides which connection to accept and which to reject. As depicted in Algorithm 2, this connection management is done by comparing the requested bandwidth from a particular user to the one that was calculated off-line in the first phase $b_{k}^{\star}$. If the requested bandwidth from the MT $k$ (i.e., $b_{k}^{(\text {Req) }}$ ), is lower than the $b_{k}^{\star}$, the connection is accepted otherwise it is rejected. In addition, at every period of time $\Delta t$ the simulator updates the REI for each accepted user using equations (29) and (32).

Finally, it was possible to improve the resources management mechanism with a third phase where some level of negotiation could happen between the MT and the network. Instead of rejecting requests with requested bandwidth higher than the efficient one, the network can negotiate an alternative lower bandwidth. This mechanism and the corresponding negotiation protocol have also been implemented in the simulator and the resulting REI have been compared for both strategies. $\overline{\text { Algorithm } 2 \text { Users connections management and rewards }}$ evaluation

Input: $\mathbf{b}^{\star}, N_{g}, N_{s}\left(N_{s}+N_{g}=N_{\max }\right)$ $\mathbf{b}^{(\mathrm{Req})}=\left[b_{1}^{(\mathrm{Req})}, \ldots, b_{N_{\max }}^{(\mathrm{Req})}\right]$ and user preferences.

Output: accepted/rejected users, and $R E I$.

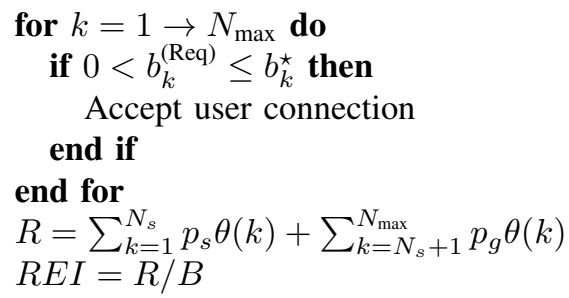

where $\theta_{g}(k)=1$ if the $k$ th user is a gold one, $\theta_{g}(k)=0$ otherwise,

and $\theta_{s}(k)=1$ if the $k$ th user is a silver one, $\theta_{g}(k)=0$ otherwise.

Table VII

RESOURCE EFFICIENCY INDEX USING MATLAB

\begin{tabular}{|c|c|c|c|c|}
\hline$\left(\mathrm{N}, C_{B W}\right)$ & $(10,400)$ & $(10,500)$ & $(20,800)$ & $(20,900)$ \\
\hline Cobb-Douglas & 0.44 & 0.441 & 0.421 & 0.443 \\
\hline Proposed Solution & 0.547 & 0.525 & 0.553 & 0.608 \\
\hline
\end{tabular}

\section{B. Results}

1) Results obtained with numerical analysis: In this section, we present the results obtained with the numerical analysis. The results are presented in Table VII, where the resource efficiency index has been provided for different values of $N$ and $C_{B W}$. This table shows that, for each value of $N$ and $C_{B W}$, our solution provides a higher index than the solution using Cobb-Douglas acceptance probability. This means that by including the preferences of the end users in the resource allocation mechanism, a network operator can improve his resource efficiency index (in our simulation scenario, this improvement is between $19 \%$ and $37 \%$ ). More precisely, by taking into account the preferences of the customers in the aggregate utility function at both the terminal side and network side, the proposed solution can satisfy not only end users but also network operators. A simple explanation of this result is that when the customers are satisfied with their experience using a particular network their willingness to use it more frequently increases. Therefore the operator revenue increases. This is a very interesting result since, unlike other existing approaches, our solution does not have a side effect on the business of operators.

2) Results obtained with the simulation platform: The results obtained with the simulation platform aims at comparing the REI of the Cobb-Douglas acceptance probability and the proposed multiplicative utility-based acceptance probability. As already mentioned, the proposed acceptance probability includes two possible scenarios, which differentiate between each other when the network cannot fulfill the requested bandwidth: i) in the "Multiplicative utility with rejection case" the running MT needs to disconnect from the network ii) in 
Table VIII

RESOURCE EFFICIENCY INDEX USING NS-2

\begin{tabular}{|c|c|c|c|c|}
\hline$\left(\mathrm{N}, C_{B W}\right)$ & $(10,400)$ & $(10,500)$ & $(20,800)$ & $(20,900)$ \\
\hline Cobb-Douglas & 0.44 & 0.441 & 0.421 & 0.443 \\
\hline Multiplicative utility without rejection & 0.70 & 0.59 & 0.60 & 0.673 \\
\hline Multiplicative utility with rejection & 0.66 & 0.48 & 0.53 & 0.635 \\
\hline
\end{tabular}

the "Multiplicative utility without rejection case" a negotiation phase between the network and the terminal occurs.

At the beginning of the simulation, all MTs initiate a connection to the $3 \mathrm{G}$ network and later they could switch to WiMAX. The Resource Efficiency Indexes of the CobbDouglas based solution and the solutions based on our two algorithms (phase 1 and phase 2) that were calculated are provided in Table VIII. It highlights that our simulations outperform the Cobb-Douglas one. Indeed, the proposed the multiplicative utility-based strategy helps the operators to improve their resource utilization by a factor of $16 \%$ and $42 \%$ versus the original Cobb-Douglas based strategy. Eventually, these results confirm also the results obtained using Matlab confirms the benefit of the proposed solution within a realistic simulation environment (MT, network infrastructure, user mobility, radio channels, etc.)

\section{Conclusion}

This paper presents a contribution in the area of network selection strategies. Next generation wireless networks will not be based on one unique "winner" technology but a smart combination of available technologies. Taking advantage of this diversity and selecting the best wireless access network in an automatic way so that to improve the quality of experience of end users is the main challenge. In this work, we have shown the importance of achieving this selection in an efficient way for both perspectives, the end user and the network operators. Given the end user preferences, which can be numerous and possibly in conflict, we have proposed a multi-criteria utility function that satisfies all the properties to maximize the quality of experience of the end user. We have also demonstrated that the proposed function can be used as an acceptance probability for the network operators' radio resource management. The suitability and the effectiveness of the proposed functions have been analyzed using numerical analysis as well as using a new multi-technologies network simulation platform. An important highlighted result is that the network access selection eventually not only benefits the end users but also increases the revenues of network operators. This result could help the wide deployment of ANS in future MTs and smartphones.

\section{REFERENCES}

[1] L. Harju and J. Nurmi, "A baseband receiver architecture for UMTSWLAN interworking applications," in Proc. of 9th ISCC, vol. 2, Egypt, 2004 , pp. $678-685$.

[2] H. J. Wang, R. H. Katz, and J. Giese, "Policy-enabled handoffs across heterogeneous wireless networks," in Proc. of the Second IEEE Workshop on Mobile Computer Systems and Applications, Washington, DC, USA, 1999, pp. 51-.
[3] L. J. Chen, T. Sun, B. Chen, V. Rajendran, and M. Gerla, "A smart decision model for vertical handoff," in 4th International Workshop on Wireless Internet and Reconfigurability, Athens, Greece, 2004, pp. 1-5.

[4] E. Adamopoulo, K. Demestichas, A. Koutsorodi, and M. Theologou, "Intelligent access network selection in heterogeneous networks - simulation results," in International Symposium on Wireless Communication Systems, 2005, pp. 279-283.

[5] O. Ormond, G. Muntean, and J. Murphy, "Utility-based intelligent network selection in beyond $3 \mathrm{G}$ systems," in IEEE International Conference on Communications, Turkey, 2006, pp. 1-6.

[6] _ _ "Economic model for cost effective network selection strategy in service oriented heterogeneous wireless network environment," in Proc. of NOMS'06, Canada, 2006, pp. 1-4.

[7] J. Chen, K. Yu, Y. Ji, and P. Zhang, "Non-cooperative distributed network resource allocation in heterogeneous wireless data networks," in Proc. of IST Mobile Summit, Greece, pp. 1-6.

[8] H. Chan, P. Fan, and Z. Cao, "A utility-based network selection scheme for multiple services in heterogeneous networks," in Proc. of International Conference on Wireless Networks, Communications and Mobile Computing, China, 2005, pp. 1175-1180.

[9] V. Gazis, N. Houssos, N. Alonistioti, and L. Merakos, "On the complexity of "always best connected" in $4 \mathrm{~g}$ mobile networks," in Proc. of IEEE Vehicular Technology Conference (VTC), Orlando, Florida, USA, 2003.

[10] R. L. Keeney and H. Raiffa, Decisions with Multiple Objectives: Preferences and Value Tradeoffs. Cambridge University Press, 1993.

[11] M. Xiao, N. Shroff, and E. Chong, "A utility-based power-control scheme in wireless cellular systems," IEEE/ACM Transactions on Networking, vol. 11, no. 2, pp. 210-221, 2003.

[12] H. Lin, M. Chatterjee, S. Das, and K. Basu, "ARC: an integrated admission and rate control framework for competitive wireless CDMA data networks using noncooperative games," IEEE Transactions on Mobile Computing, vol. 4, no. 3, pp. 243-258, 2005.

[13] L. Badia, M. Lindstrom, J. Zander, and M. Zorzi, "An economic model for the radio resource management in multimedia wireless systems," Computer Communications, vol. 27, no. 11, pp. 1056-1064, 2004.

[14] X. Yan, Y. A. ekerciolu, and S. Narayanan, "A survey of vertical handover decision algorithms in fourth generation heterogeneous wireless networks," Computer Networks, vol. 54, no. 11, pp. 1848 - 1863, 2010.

[15] "An overview of vertical handover techniques: Algorithms, protocols and tools," Computer Communications, vol. 34, no. 8, pp. 985 - 997, 2011.

[16] S. Lee, K. Sriram, K. Kim, Y. H. Kim, and N. Golmie, "Vertical handoff decision algorithms for providing optimized performance in heterogeneous wireless networks," IEEE Transactions on Vehicular Technology, vol. 58, no. 2, pp. 865 -881, 2009.

[17] H. Marques, J. Ribeiro, P. Marques, and J. Rodriguez, "Simulation of 802.21 handovers using ns-2," J. Comp. Systems Networks Communications, vol. 2010, pp. 1-7, 2010.

[18] J.-S. Wu, S.-F. Yang, and B.-J. Hwang, "A terminal-controlled vertical handover decision scheme in IEEE 802.21-enabled heterogeneous wireless networks," International Journal on Communication Systems, vol. 22, no. 7, pp. $819-834,2009$.

[19] V. Sharma, A. Agarwal, and M. Qadeer, "Media independent handover (IEEE 802.21): Framework for next generation vertical handover protocols," in International Conference on Computational Intelligence and Communication Networks (CICN), India, 2011, pp. 507 -511.

[20] S.-J. Wu and C.-Y. Yang, "Hip-based handover mechanism under MIH architecture in heterogeneous wireless networks," in 7th International Conference on Networked Computing and Advanced Information Management (NCM), Gyeongju, Korea, 2011, pp. 329 -334.

[21] Y. Zhang, W. Zhuang, and A. Saleh, "Vertical handoff between 802.11 and 802.16 wireless access networks," in Proc. of IEEE Global Telecommunications Conference (GLOBECOM)'08, New Orleans, USA, 2008, pp. 584-589. 
[22] Q. Mussabbir, W. Yao, Z. Niu, and X. Fu, "Optimized FMIPv6 using IEEE 802.21 MIH services in vehicular networks," IEEE Transactions on Vehicular Technology, vol. 56, no. 6, pp. 3397 -3407, 2007.

[23] S. Fernandes and A. Karmouch, "Vertical mobility management architectures in wireless networks: A comprehensive survey and future directions," IEEE Surveys Tutorials on Communications, vol. 14, no. 1, pp. $45-63,2012$.

[24] A. Yoon-Young and J. Junghoon, "Multi-radio power management consideration," presented at IEEE 802.21, Session No.23, 2007.

[25] Q. T. Nguyen-Vuong, N. Agoulmine, and Y. Ghamri-Doudane, "Terminal-controlled mobility management in heterogeneous wireless networks," IEEE Communication Magazine, vol. 45, no. 4, pp. 122129, 2007.

[26] Q. Nguyen-Vuong, Y. Ghamri-Doudane, and N. Agoulmine, "On utility models for access network selection in wireless heterogeneous networks," in Proc. of IEEE/IFIP Network Operations and Management Symposium - NOMS 2008, Brazil, 2008.

[27] E. H. Cherkaoui, N. Agoulmine, T. Nguyen, L. Toni, and J.-G. Fontaine, "Taking advantage of the diversity in wireless access networks: On the simulation of a user centric approach," in Integrated Network Management (IM)'2011, Ireland, 2011, pp. 1021-1028.

[28] L. Giupponi, R. Agusti, J. Prez-Romero, and O. Sallent, "Towards balancing user satisfaction and operator revenue in beyond 3G cognitive networks," in Proceedings of the 15th IST Mobile and Wireless Summit, Greece, 2006, pp. 1-5.

[29] N. N. Ahmed Hasswa and H. Hassanein, "Generic vertical handoff decision function for heterogeneous wireless networks," in IFIP Conference on Wireless and Optical Communications, Dubai, 2005, pp. 239-243.

[30] L. Badia, M. Lindström, J. Zander, and M. Zorzi, "Demand and pricing effects on the radio resource allocation of multimedia communication systems," in Proc. of IEEE Global Telecommunications Conference (GLOBECOM)'03, vol. 7, San Francisco, USA, 2003, pp. 4116 - 4121

[31] X. Bai, L. Boloni, D. Marinescu, H. J. Siegel, R. Daley, and I.-J. Wang, "Are Utility, Price, and Satisfaction Based Resource Allocation Models Suitable for Large-Scale Distributed Systems?" in Proc. of the 3rd International Workshop on Grid Economics and Business Models (GECON 2006), Singapore, May 2006, pp. 113-122.

[32] "MIH-NIST-NS2.29-LTE: an ns2 path for multi criteria network making decision, 2012." [Online]. Available: http://sourceforge.net/projects/mihnistns229/files/latest/download

[33] "The Network Simulator NS-2," http://www.isi.edu/nsnam/ns/.

[34] The Network Simulator NS-2 NIST add-on IEEE 802.21 model based on IEEE P802.21/D03.00, NIST, January 2007.

[35] G. Piro, L. A. Grieco, G. Boggia, F. Capozzi, and P. Camarda, "Simulating lte cellular systems: an open source framework," IEEE Transactions on Vehicular Technology, vol. 60, pp. 498-513, 2011.

[36] J. Tian, J. Hähner, C. Becker, I. Stepanov, and K. Rothermel, "Graphbased mobility model for mobile ad hoc network simulation," in Proc. of the 35th Annual Simulation Symposium, San Diego, California, 2002, pp. 337-345.

[37] K. Jones and J. Ousterhout, TCL and the Toolkit. Addison-Wesley Educational Publishers, 2009.

[38] O. Oyman and S. Singh, "Quality of experience for http adaptive streaming services," IEEE Communications Magazine, vol. 50, no. 4 pp. 20-27, 2012.

[39] M. P. Michael, "Energy awareness for mobile devices," in Researh Seminar on Energy Awareness, University of Helsinki, Helsinki, 2005.

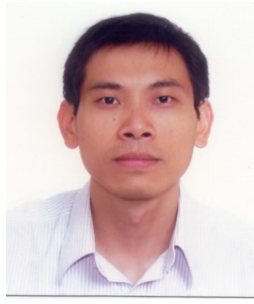

Q.T. Nguyen-Vuong is currently working at the Networks and Services division at France Telecom. Prior to joining France Telecom, he spent almost three years as a 3G IP RAN expert at Davidson Consulting. He received his masters degree from University of Paris 6 and his PhD from the University of Evry (France) in computer networks respectively in 2005 and 2008. He received the engineering diplomas of the Ecole Polytechnique (France) and Telecom ParisTech (France) in 2005. His research interests include the resource management in wireless network, mobility management, fixed and mobile service convergence, all-IP mobile network management and IMS-based rich unified communication.

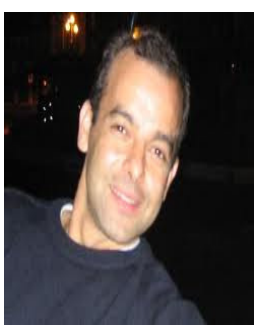

Nazim AGOULMINE is a Senior IEEE member. $\mathrm{He}$ is a full professor at the University of Evry since 2000 (France) and the head of the Research Programme on Software and Hardware Infrastructures for Future ICT at ANR (National French Research Funding Agency). He is leading a the research group LRSM (Software for Networked Multimedia Systems) as part of the IBISC Research Laboratory. He received his Engineer degree in 1988 from USTHB, Algeria and his Master and Phd degrees in computer science respectively in 1989 and 1992 from the University of Paris XI, France. His research interests are mainly wired and integrated network and service management, autonomic systems, wire and wireless networks, ehealth, bio-inspired networking and computing. He is an area editor of the Elseviers International Journal on Computer Networks and the secretary of the ehealth IEEE Technical Committee. He served several times as a general TPC co-chair for IEEE/IFIP conferences and workshops (IM, GIIS, MMNS, DANSM, LANOMS, etc.). He is author and co-author of five books in the areas of autonomic networking, self-managed networks network and system management and multimedia management. He has participated in numerous European projects from the first EU programs (RACE ESPRIT, ACTS, IST, FP, ITEA, CELTIC) He is acting as an expert for several national and international research agencies ANR (France), NSERC/FCAR (Canada), HETAC (Ireland), NRK (South Korea)

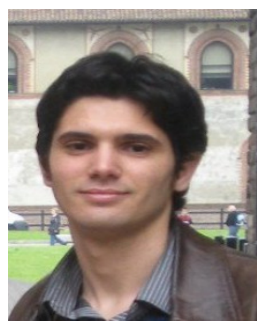

El Hadi Cherkaoui is currently a Ph.D. student at The Italian Institute of Technology (IIT) and University of Genova (Italy). He received his Master degree in Computer Science from the University of Science and Technics of Montpellier II (France), in September 2009. From April 2009 to December 2009, he had been collaborating with the IBISC/LRSM Laboratory at the University of Evry (France), focusing his research activity on seamless mobility and access selection in heterogeneous wireless networks. During 2012, he was a visiting scholar at the SIGNET Laboratory, University of Padova (Italy), working on packet error recovery schemes in underwater networks. His research interests include the design and optimization of vertical and horizontal handovers in 4G heterogeneous networks, underwater communications and Wireless Sensor Networks (WSNs). He is member of IEEE.

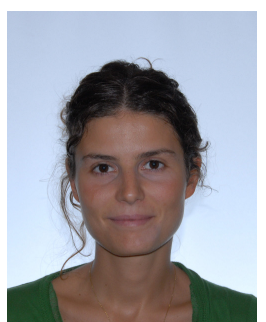

Laura Toni (S'06-M'09) received the M.S. degree (with honors) in electrical engineering and the Ph.D. degree in electronics, computer science and telecommunications from the University of Bologna, Italy, in 2005 and 2009, respectively.

In 2005, she joined the Department of Electronics, Informatics and Systems at the University of Bologna, to develop her research activity in the area of wireless communications. During 2007, she was a visiting scholar at the University of California at San Diego, CA, working on video processing over wireless systems. Since 2009, she has been a frequent visitor to the UCSD, working on joint source and channel coding for wireless communication systems.

From June 2009 to November 2011, she worked at the Tele-Robotics and Application (TERA) department at the Italian Institute of Technology (IIT) as a Post-doctoral Fellow. From November 2011 to November 2012, she had been a Post-doctoral fellow in the Electrical and Computer Engineering department at the University of California, San Diego. Since December 2012, she has been Post-doctoral fellow in the Signal Processing Laboratory (LTS4) at the École Polytechnique Fédérale de Lausanne (EPFL), Switzerland. Her research interests are in the areas of image and video processing, wireless communications, and underwater communications. 